\title{
Tensile Deformation Behavior of Duplex Stainless Steel Studied by In-Situ Time-of-Flight Neutron Diffraction
}

\begin{abstract}
N. JIA, R. LIN PENG, D.W. BROWN, B. CLAUSEN, and Y.D. WANG
For a duplex alloy being subjected to deformation, the different mechanical behaviors of its constituent phases may lead to a nonuniform partition of stresses between phases. In addition, the grain-orientation-dependent elastic/plastic anisotropy in each phase may cause grain-tograin interactions, which further modify the microscopic load partitioning between phases. In the current work, neutron diffraction experiments on the spectrometer for materials research at temperature and stress (SMARTS) were performed on an austenite-ferrite stainless steel for tracing the evolution of various microstresses during tensile loading, with particular emphasis on the load sharing among grains with different crystallographic orientations. The anisotropic elastic/plastic properties of the duplex steel were simulated using a visco-plastic self-consistent (VPSC) model that can predict the phase stress and the grain-orientation-dependent stress. Material parameters used for describing the constitutive laws of each phase were determined from the measured lattice strain distributions for different diffraction $\{h k l\}$ planes as well as the laboratorial macroscopic stress-strain curve of the duplex steel. The present investigations provide in-depth understanding of the anisotropic micromechanical behavior of the duplex steel during tensile deformation.
\end{abstract}

DOI: $10.1007 / \mathrm{s} 11661-008-9675-2$

(C) The Minerals, Metals \& Materials Society and ASM International 2008

\section{INTRODUCTION}

So far, duplex stainless steels with a microstructure consisting of approximately equal volumes of austenite and ferrite have been widely applied under various environments, owing to their excellent mechanical properties and high resistance to stress corrosion cracking. The attractive mechanical properties of such materials are obtained mainly by the combined effects of ferrite on raising the yield strength and austenite with a high strain hardening on favoring the tensile strength. ${ }^{[1]}$ Accordingly, the respective different mechanical properties and the elastic/plastic anisotropy of each constituent phase may lead to two kinds of microstresses in the duplex steel, including the phase stress and intergranular stress (or grain-orientation-dependent stress). The former is produced by the phase-to-phase interactions and the latter is due to the grain-to-grain interactions. ${ }^{[2,3]}$ The presence of the internal microstresses is closely related to the evolution of the macrostress subjected in

N. JIA, Lecturer, and Y.D. WANG, Professor, are with Key Laboratory for Anisotropy and Texture of Materials, Ministry of Education, Northeastern University, Shenyang 110004, China. Contact email: nanjia_neu@yahoo.com.cn R. LIN PENG, Associate Professor, is with Department of Mechanical Engineering, Linköping University, S-58183 Linköping, Sweden. D.W. BROWN and B. CLAUSEN, Facility Scientists, are with Los Alamos Neutron Scattering Center, Los Alamos National Laboratory, Los Alamos, NM 87545.

This article is based on a presentation given in the symposium entitled "Neutron and X-Ray Studies for Probing Materials Behavior," which occurred during the TMS Spring Meeting in New Orleans, LA, March 9-13, 2008, under the auspices of the National Science Foundation, TMS, the TMS Structural Materials Division, and the TMS Advanced Characterization, Testing, and Simulation Committee. Article published online October 10, 2008 service. Both macrostresses and microstresses greatly affect the performance of engineering components. Therefore, in recent years, great effort has been made toward exploring a framework within which the information regarding various internal stresses can be incorporated into the design of materials used in various critical industries. ${ }^{[-6]}$

The neutron diffraction technique is a well established tool for studying micromechanical behavior of materials. ${ }^{[7,8]}$ Because of the selective nature of diffraction techniques, the elastic (lattice) strain of the specified grain populations of each phase with their $(h k l)$ crystal planes along the macroscopic specimen directions may be experimentally determined, which enables us to separate the elastic response of different phases during deformation. On the other hand, the self-consistent models prove to be effective in describing the anisotropic micromechanical behaviors of single-phase materials during elastic and plastic deformation. ${ }^{[9,10]}$ For twophase materials, however, the interactions of grain-tograin and phase-to-phase may occur simultaneously during deformation, which requires a complicated model for describing the micromechanical behavior. ${ }^{[11-13]}$ In the present work, based on time-of-flight (TOF) neutron diffraction experiments, different lattice strains in an austenite-ferrite $(\gamma-\alpha)$ stainless steel, SAF 2507 , during uniaxial tensile deformation were measured. Since multiple reflections of different phases had been captured simultaneously, material parameters required for the numerical modeling of heterogeneous stresses within the material can be determined from the experimental lattice strain distributions. A visco-plastic selfconsistent (VPSC) model developed for two-phase materials ${ }^{[14]}$ was then implemented for simulating both 
the grain-orientation-dependent stress and the phase stress in the duplex steel. A quantitative and accurate evaluation of the partitioning of stress in the duplex stainless steel is achieved and the developed VPSC model for two-phase materials is further verified.

\section{IN-SITU NEUTRON DIFFRACTION EXPERIMENTS}

\section{A. Materials and Experimental Procedures}

A commercial superduplex stainless steel, SAF 2507, provided by Sandvik AB (Sandviken, Sweden) was used in this study. The nominal chemical composition of the steel is (wt pct): $25 \mathrm{Cr}, 7 \mathrm{Ni}, 4 \mathrm{Mo}, 1.2 \mathrm{Mn}, 0.8 \mathrm{Si}$, and $0.3 \mathrm{~N}$ (remainder Fe). The as-received hot-rolled bar of $20 \mathrm{~mm}$ in diameter was supplied in solution-annealed and water-quenched condition. By applying the electron back-scattering diffraction (EBSD) technique on different sections of the material, ${ }^{[15]}$ the phase composition consisting of $58 \mathrm{vol}$ pct austenite $(\gamma)$ and $42 \mathrm{vol}$ pct ferrite $(\alpha)$ (in volume) is identified. The microstructure is characterized by a columnar shape of grains with an aspect ratio of approximate 1:1:3 (ND:TD:RD) in both phases, where RD is the rolling direction, and transverse direction (TD) and normal direction (ND) denote the two radial directions perpendicular to each other. The initial textures in this material have also been previously revealed by performing X-ray diffraction experiments on the $\gamma$ and $\alpha$ phases. ${ }^{[15]}$ The 0.2 pct offset yield strength and fracture tensile ductility of the duplex steel are $606 \mathrm{MPa}$ and 30 pet, respectively.

A cylindrical specimen of $6-\mathrm{mm}$ diameter $\times 44-\mathrm{mm}$ gage length was machined along the axis of the as-received bar material so that the loading direction (LD) was parallel to the RD for the neutron diffraction experiments. In order to remove residual stresses induced by the mechanical processing and the previous thermal treatment, the specimen was then annealed at $550{ }^{\circ} \mathrm{C}$ for 1.5 hours in a vacuum furnace and immediately water quenched to room temperature.

For the in-situ loading measurements, the spectrometer for materials research at temperature and stress (SMARTS $^{[16]}$ at Los Alamos National Laboratory was used. The specimen was loaded incrementally from $5 \mathrm{MPa}$ into the plastic regime and reached $800 \mathrm{MPa}$, then unloaded to $400 \mathrm{MPa}$, and finally to $5 \mathrm{MPa}$. At different loading levels, the TOF neutron spectra were collected by the two detector banks at fixed scattering angles. As shown in the schematic of the SMARTS diffractometer (Figure 1), the two banks of detectors are arranged such that the lattice planes with the normal direction perpendicular to the loading axis are measured by bank 1 and the lattice planes with the normal direction parallel to the loading axis are measured by bank 2. Considering that the TOF is proportional to the neutron wavelength, i.e.,

$$
\lambda=h t /(m L)
$$

where $h$ is Planck's constant, $m$ is the neutron mass, and $L$ is the traveling path of neutrons, we obtain the

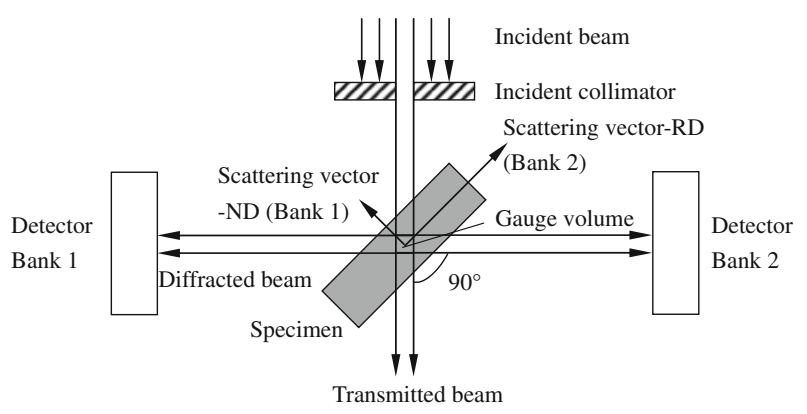

Fig. 1-Schematic of SMARTS diffractometer for stress mapping. Incident and diffracted beams form the gage volume. Tensile loading is applied along the RD.

interplanar spacing, $d_{h k l}$, of the $h k l$ plane in terms of Bragg's law; i.e.,

$$
d_{h k l}=\lambda_{h k l} /\left(2 \sin \theta_{h k l}\right)=h t_{h k l} /\left(2 m L \sin \theta_{h k l}\right)
$$

where $2 \theta_{h k l}$ is the diffraction angle. The TOF, $t_{h k l}$, of the $h k l$ plane was determined by fitting individual peaks in the diffraction spectra by the GSAS software. ${ }^{[17]}$ For the investigated duplex steel, the $\{111\} \gamma$, $\{200\} \gamma,\{220\} \gamma$, and $\{311\} \gamma$ reflections of austenite and the $\{110\} \alpha,\{200\} \alpha$, and $\{211\} \alpha$ reflections of ferrite were analyzed. Then the lattice strain $\varepsilon_{h k l}$ was calculated as

$$
\varepsilon_{h k l}=\left(d_{h k l}-d_{0}\right) / d_{h k l}=\left(t_{h k l}-t_{0}\right) / t_{h k l}
$$

where $d_{0}$ and $t_{0}$ denote the interplanar spacing and the neutron TOF for the specified $h \mathrm{kl}$ plane of a stress-free material, respectively. In the current work, the measurement at the initial load of $5 \mathrm{MPa}$ was taken as the stress-free state of the specimen, which means that phase stresses due to thermal mismatch are not considered in the current analysis.

\section{B. Experimental Results}

The anisotropic response of lattice strain for different reflections to the applied stress is important for verifying the micromechanical model (VPSC) to be used in this study. Figures 2 and 3 show the evolution of lattice strains, $\varepsilon_{h k l}$, for different $h k l$ planes in $\gamma$ and $\alpha$ phases as a function of the applied tensile stress, parallel and perpendicular to the LD, respectively. The simulated results are also displayed as solid lines for different $h k l$ planes, which will be further discussed in Section IV. As shown in Figure 2, an almost linear response of lattice strain to applied stress is observed for all the investigated $h k l$ planes before loading up to $450 \mathrm{MPa}$, which indicates that the elastic behavior is dominant during this stage in both phases. Different slopes for the different $h \mathrm{kl}$ planes are mainly associated with the respective diffraction elastic modulus. When the loading is above $600 \mathrm{MPa}$, deviations from the elastic linear response occur in the lattice strains for most $h k l$ planes in austenite and ferrite, signifying the onset of macroscopic plasticity in both phases. In the plastic region as displayed in Figure 2, the nonlinearity starts at different 


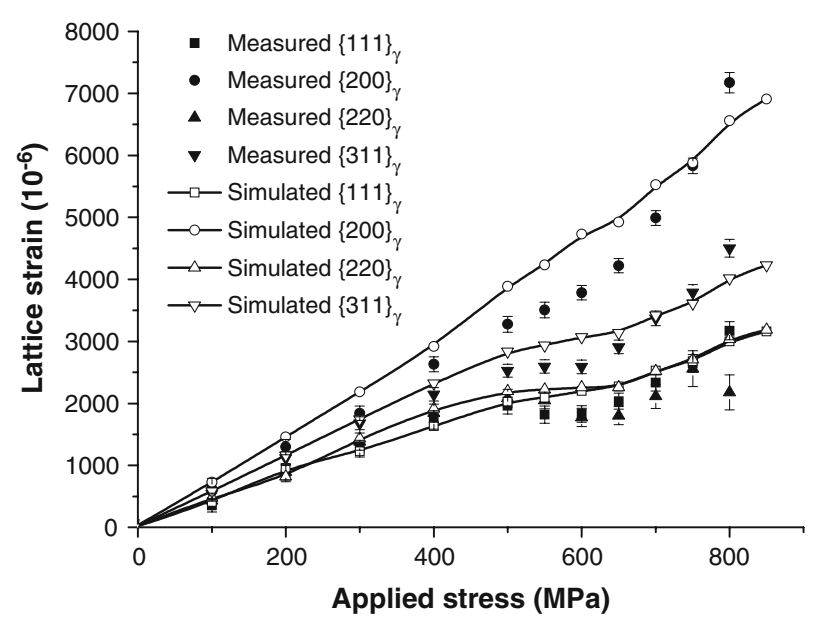

(a)

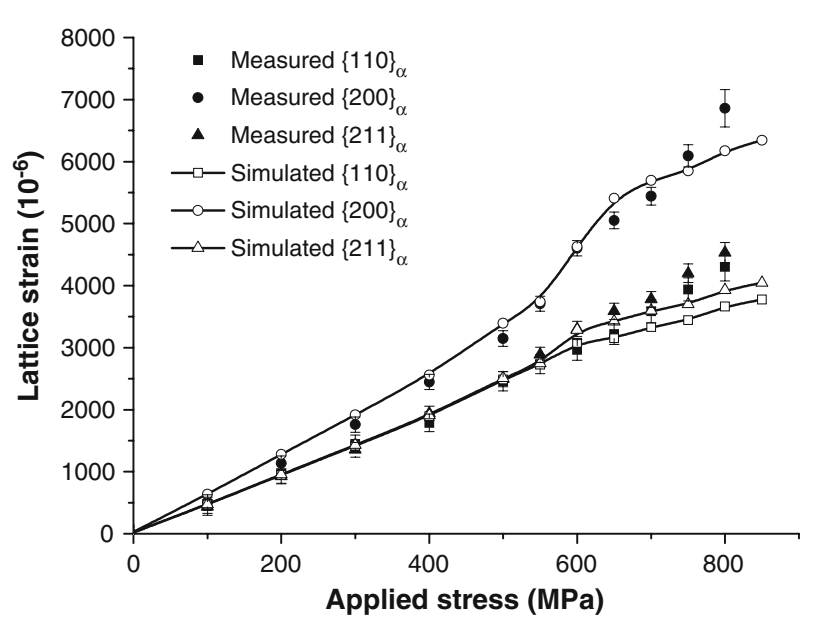

(b)

Fig. 2-Measured and simulated response of lattice strains to applied stress parallel to the LD for different reflections in $(a)$ austenite and $(b)$ ferrite.

macrostresses for different grain orientations and the slope varies among them, indicating the different yielding behaviors of different grain populations in each phase. It can be seen that for the austenite the $\{220\}$ and $\{311\}$ grains yield at about $450 \mathrm{MPa}$, and the $\{111\}$ and $\{200\}$ grains do not show a deviation from the linearity until the load of $500 \mathrm{MPa}$ is reached. For the ferrite, the $\{211\}$ grains yield preferentially $(\sim 600 \mathrm{MPa})$ compared with the grains with $\{110\}$ and $\{200\}$ orientations ( $650 \mathrm{MPa})$. These observations are in agreement with our previous investigations on the deformation behavior of the same material under compressive loading. ${ }^{[15]}$ Concerning measurements in the TD (perpendicular to the LD) as shown in Figure 3, the following features are captured: at the initial stage of deformation, all the lattice reflections show an elastic linear response with a slope in opposite sign to that found along the axial direction (parallel to the LD); and when the plasticity starts in the macrostress-strain curve $(\sim 500 \mathrm{MPa})$, the nonlinear response of lattice strain becomes evidenced for all the reflections and some reflections start to show more tensile strains, which is consistent with

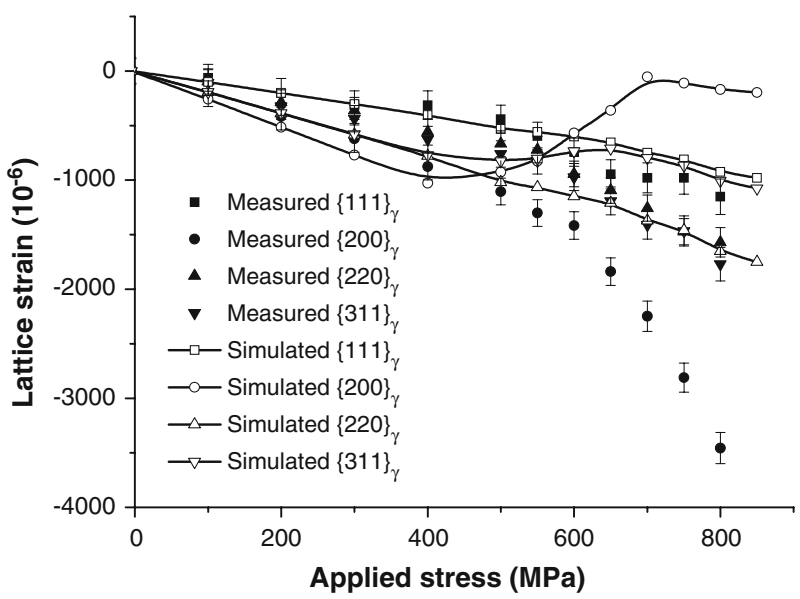

(a)

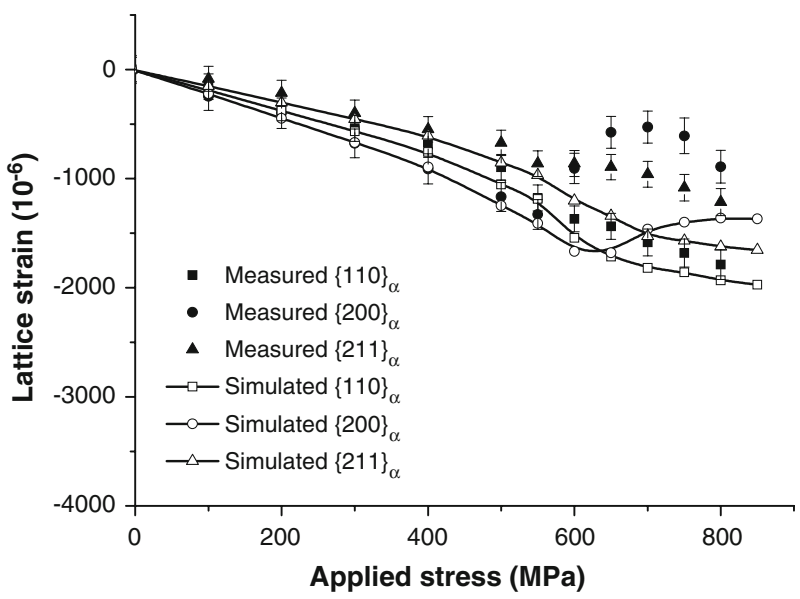

(b)

Fig. 3-Measured and simulated response of lattice strains to applied stress perpendicular to the LD for different reflections in (a) austenite and (b) ferrite.

observations in the axial direction. For one specific population of grains, the nonlinearity in the responses of lattice strains along the two specimen directions, i.e., the axial direction and the TD, was observed at different macrostresses. This can be attributed to the effect of diffraction Poisson's ratio for each $h k l$ lattice plane.

\section{SELF-CONSISTENT MODEL}

A VPSC model was implemented for simulating the micromechanical behavior of the studied duplex steel during tensile test. In this model, several thousands of grains with their own Euler angles are constructed for each phase to provide the information on grain orientations and volume fractions, which is in agreement with the initial textures of the investigated material. Each grain is treated as an elastoplastic inclusion with instantaneous moduli $L_{c}$ in a homogeneous equivalent medium. Then under the boundary condition that the external loading is applied incrementally up to the targeted macroscopic stress, the scheme includes 
elastic-plastic interactions among all those grains in the polycrystal with the overall moduli $L$ of the duplex steel. In terms of Eshelby's observation, ${ }^{[18]}$ the stress and strain rates in the ellipsoidal inclusion $\dot{\varepsilon}_{c}$ is uniform and thus can be related to the strain rates at infinity by a fourth-order tensor $A_{c}$ according to $\dot{\varepsilon}_{c}=A_{c} \dot{\bar{\varepsilon}}$. Then for the $n$th phase in the duplex steel, the interaction equation that links microscopic stress-strain states at the grain scale $\left(\dot{\varepsilon}_{c}^{(n)}\right)$ to the macroscopic state at the polycrystal scale $(\dot{\bar{\varepsilon}})$ is derived in an iterative way by

$$
\dot{\varepsilon}_{c}^{(n)}=A_{c}^{(n)} \dot{\bar{\varepsilon}}
$$

with $A_{c}^{(n)}=\left(L^{*}+L_{c}^{(n)}\right)^{-1}\left(L^{*}+L\right)$, where $L^{*}$ is introduced as a "constraint" tensor for a matrix containing an ellipsoidal void with the same orientation and shape as the grain. ${ }^{[19]}$

To simulate the loading process, the model is governed by the single-crystal constitutive law that considers just slip mechanisms. For austenitic phase with the fcc crystal structure, slip on the close-packed $\{111\}$ planes and in the $\langle 110\rangle$ close-packed directions is considered. For ferritic phase with the bcc crystal structure, $\{110\}\langle 111\rangle$ and $\{112\}\langle 111\rangle$ are considered. Then for each single crystal, the hardening function of its $i$ th slip system is described by ${ }^{[20]}$

$$
\tau^{i}(\Gamma)=\tau_{0}^{i}+\left(\tau_{1}^{i}+\theta_{1}^{i} \Gamma\right)\left[1-\exp \left(-\frac{\theta_{0}^{i} \cdot \Gamma}{\tau_{1}^{i}}\right)\right]
$$

where $\Gamma$ is the shear increment accumulated on all systems over the deformation history and $\tau^{i}$ is the instantaneous flow stress as a function of $\Gamma$. As explained in Reference 21 and illustrated in Figure 4, the initial deformation is characterized by $\tau_{0}^{i}$ (the critical resolved shear stress) and $\theta_{0}^{i}$ (the hardening rate), while the asymptotic hardening is characterized by $\tau_{1}^{i}$ and $\theta_{1}^{i}$. Therefore, the material parameters are determined by fitting the neutron diffraction measured lattice strain distributions as well as the laboratorial macrostressstrain curve of the material, so as to provide fundamental inputs required for modeling the mechanical processing (as listed in Table I). The preceding singlecrystal behavior is assumed to apply to each individual grain. In addition, for the overall stress-strain behavior of the polycrystalline material, stress/strain boundary conditions imposed in the model are as follows: the strain increment (displacement gradients) of grains is inhomogeneous within the polycrystalline aggregate, and furthermore, the macroscopic properties of the polycrystal are the volume averages over the counterparts of its constituent single crystals. In the final step of self-consistent calculation, the overall stress and strain rates of various polycrystalline aggregates are calculated as

$$
\dot{\bar{\sigma}}=\left\{\dot{\sigma}_{c}\right\} \quad \text { and } \quad \dot{\bar{\varepsilon}}=\left\{\dot{\varepsilon}_{c}\right\}
$$

where \{\} denote the average over all the constituent single crystals within the polycrystal.

In this work, calculations are performed on 2592 and 1877 ellipsoidal inclusions with the axes ratio of $1: 1: 3$

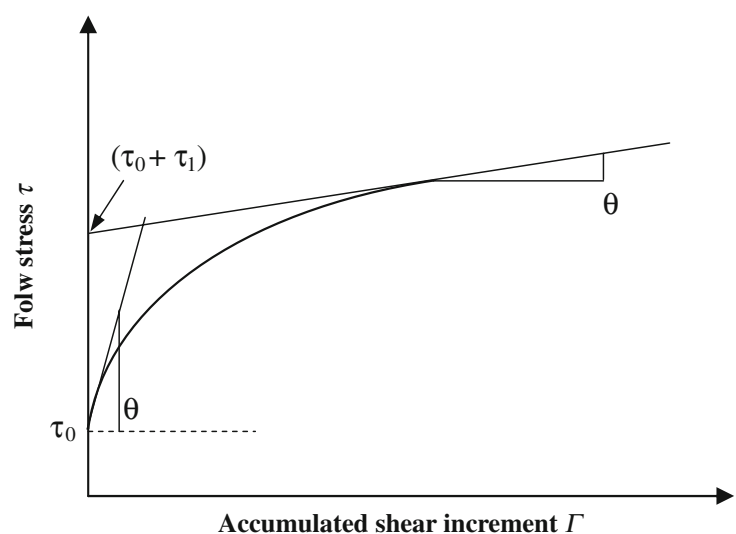

(a)

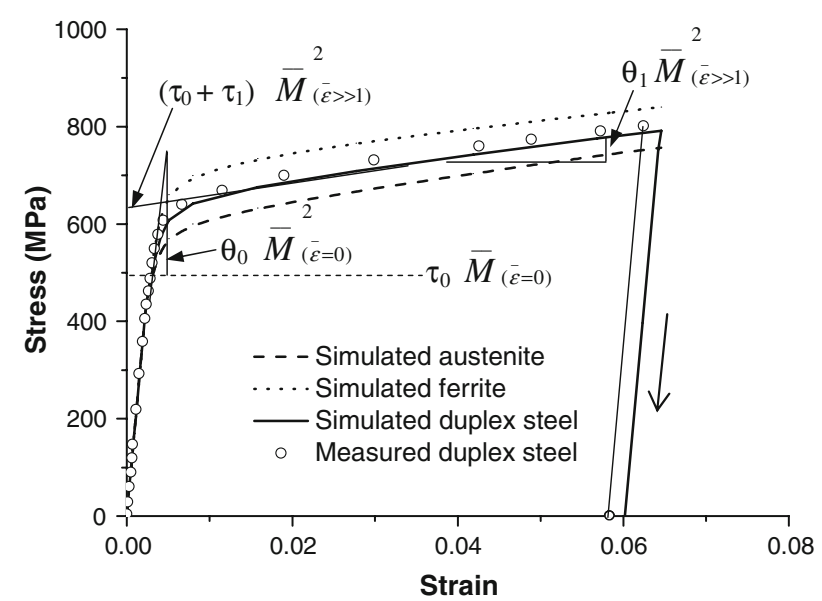

(b)

Fig. 4 - (a) Schematic representation of critical stresses and hardening parameters used in the hardening law (Eq. [5]) for each single crystal. (b) Measured and simulated stress-strain curve of the studied duplex steel and its constitutive phases. Arrow direction is shown for the unloading curve. Relationship between material parameters and macromechanical behaviors of duplex steel is indicated in (b). The term $M$ denotes the Taylor factor as a function of accumulated shear increment $(\Gamma)$ and macrostrain $(\varepsilon)$. The bar denotes averaging over all constituent single crystals.

Table I. Material Parameters of the $\gamma$ and $\alpha$ Phases Used in the Visco-Plastic Self-Consistent Simulations

\begin{tabular}{lccccc}
\hline Phases & $\begin{array}{c}\text { Slip } \\
\text { Systems }\end{array}$ & $\begin{array}{c}\tau_{0} \\
(\mathrm{MPa})\end{array}$ & $\begin{array}{c}\tau_{1} \\
(\mathrm{MPa})\end{array}$ & $\begin{array}{c}\theta_{0} \\
(\mathrm{MPa})\end{array}$ & $\begin{array}{c}\theta_{1} \\
(\mathrm{MPa})\end{array}$ \\
\hline Austenite $(\gamma)$ & $\{111\}\langle 110\rangle$ & 180 & 1 & 1200 & 800 \\
Ferrite $(\alpha)$ & $\{110\}\langle 111\rangle$ & 300 & 1 & 800 & 535 \\
& $\{112\}\langle 111\rangle$ & 320 & 1 & 800 & 535 \\
\hline
\end{tabular}

Note: $\tau$ and $\theta$ denote the critical resolved stress and the hardening parameter, respectively.

corresponding to ND, TD, and RD. The two groups of inclusions represent grains of austenite (58 pct) and ferrite (42 pct) in the duplex steel, respectively. Initial orientations of the crystallites corresponding to the experimental textures, together with the texture evolution in both phases are involved in modeling the tensile deformation. Regarding the in-situ diffraction 
experiments, since the residual macrostress within the specimen has been eliminated before testing and the data analysis was made by taking the first measurement prior to loading as the stress-free state, initial residual stress was neglected in the present simulation.

\section{PREDICTION OF MICROMECHANICAL BEHAVIOR AND DISCUSSION}

As shown in Figure 2, with the determined material parameters used in the model, the response of lattice strains to the applied stress along the LD are well captured in both phases by the simulations. This verifies the reliability of the present two-phase VPSC model for predicting the micromechanical behavior of anisotropic duplex materials. During the initial stage of deformation, different slopes that should correspond to the different elastic moduli of respective reflections are well predicted. With increasing loading, deviation from an elastic linear response caused by the preferential yielding of some grains with their specific orientations is predicted below the macroscopic plasticity for the austenite $(\sim 450 \mathrm{MPa})$, and close to the onset of macroscopic yielding for the ferrite $(\sim 600 \mathrm{MPa})$, respectively. Owing to the fact that the stress redistribution induced by both interactions of grain-to-grain within one phase and phase-to-phase has been updated at each interaction after entering into the plastic region, the preceding simulations agree with the experimental results. In the TD (Figure 3), the reproduced elastic/plastic behaviors of most lattice reflections are in good agreement with the experiments. However, large discrepancies are found in some reflections, in particular the $\{200\}$ reflections for both phases. This could be attributed to the following facts. First, the imprecise measurements of the lattice strains were induced by the decreasing Bragg intensity and the broadening diffraction peak as observed for those lattice planes when the duplex steel entered into the elastoplastic region (at above $450 \mathrm{MPa}$ ). Second, with the elevated load beyond the macroscopic yield point (at above $600 \mathrm{MPa}$ ), an increasing magnitude in creep was observed in the specimen. The negligence of creep in the simulation may contribute to the observed discrepancy.

Finally, when comparing with LD, the larger difference between the simulation and measurements in TD suggests a significant influence of texture and morphology of grains in some particular sample directions. This texture-dependent lattice strain variation of the as-received bar material was once revealed by applying the conventional $\theta / 2 \theta$ neutron scanning along its radial direction, ${ }^{[22]}$ indicating that the response of lattice strain to the applied stress include not only the elastic and plastic anisotropy among the different grain orientations, but also the effect of preferred grain orientation distribution (texture development). However, the quantitative explanation of the deformation texture under the effect of heterogeneous stress and its impact on the micromechanical behavior of the material is beyond the scope of this article.

To examine the stress distribution within the duplex steel during loading and unloading, the two-phase VPSC model was used to simulate the overall stressstrain responses for respective phases and the average stresses for specifically oriented grains along the LD, i.e., $\langle u v w\rangle / / \mathrm{LD}$ are presented in Table II. The simulation assumes that there is no internal stress within the material before loading, indicative that the initial microstresses are zero in both phases. See the $\left|\sigma_{33}^{\max }-\sigma_{33}^{\min }\right|$ values in Table II, in the elastic regime when the applied load is below $500 \mathrm{MPa}$, the intergranular microstresses in both phases evolve with the elevated load. This is due to the elastic anisotropy of different lattice reflections in individual phases. The phase stress, i.e., the $\sigma_{33}^{p}$ value, remains a very low level

Table II. Simulated Average Stress $\sigma_{33}$ and Plastic Strain $\varepsilon_{33}^{p l}$ for $\gamma$ and $\alpha$ Phases and Grain Families Oriented with $\langle u v w\rangle / /$ LD

\begin{tabular}{|c|c|c|c|c|c|c|c|c|}
\hline \multirow[b]{2}{*}{ Applied Stress } & \multicolumn{6}{|c|}{ Tensile Loading } & \multicolumn{2}{|c|}{ Unloading } \\
\hline & 100 & 400 & 500 & 600 & 700 & 800 & 400 & 0 \\
\hline$\sigma_{33}$ in $\gamma$ phase & 102 & 408 & 495 & 570 & 668 & 777 & 379 & 0 \\
\hline$\sigma_{33}$ for $\langle 100\rangle_{\gamma} / / \mathrm{LD}$ & 84 & 334 & 431 & 511 & 594 & 692 & 362 & 48 \\
\hline$\sigma_{33}$ for $\langle 110\rangle_{\gamma} / / \mathrm{LD}$ & 108 & 434 & 528 & 615 & 747 & 875 & 452 & 50 \\
\hline$\sigma_{33}$ for $\langle 111\rangle_{\gamma} / / \mathrm{LD}$ & 115 & 462 & 578 & 649 & 747 & 878 & 430 & 3 \\
\hline$\sigma_{33}$ for $\langle 311\rangle_{\gamma} / / \mathrm{LD}$ & 98 & 393 & 486 & 558 & 643 & 746 & 363 & -3 \\
\hline$\varepsilon_{33}^{p l}$ in $\gamma$ phase (pct) & 0 & 0 & 0.07 & 0.28 & 2.5 & 6.72 & 5.97 & 5.97 \\
\hline$\sigma_{33}$ in $\alpha$ phase & 102 & 408 & 526 & 660 & 766 & 855 & 420 & 0 \\
\hline$\sigma_{33}$ for $\langle 100\rangle_{\alpha} / / \mathrm{LD}$ & 93 & 372 & 479 & 616 & 733 & 813 & 413 & 33 \\
\hline$\sigma_{33}$ for $\langle 110\rangle_{\alpha} / / \mathrm{LD}$ & 109 & 437 & 564 & 696 & 827 & 944 & 478 & 26 \\
\hline$\sigma_{33}$ for $\langle 111\rangle_{\alpha} / / \mathrm{LD}$ & 114 & 457 & 590 & 752 & 905 & 1005 & 516 & 43 \\
\hline$\sigma_{33}$ for $\langle 211\rangle_{\alpha} / / \mathrm{LD}$ & 109 & 436 & 562 & 713 & 821 & 912 & 445 & -5 \\
\hline$\varepsilon_{33}^{p l}$ in $\alpha$ phase $(\mathrm{pct})$ & 0 & 0 & 0 & 0.05 & 2.25 & 7.00 & 6.09 & 6.09 \\
\hline$\sigma_{33}^{p}$ phase stress & 0 & 0 & 31 & 90 & 98 & 78 & 41 & 0 \\
\hline$\left|\sigma_{33}^{\max }-\sigma_{33}^{\min }\right|$ in $\gamma$ phase & 34 & 128 & 147 & 138 & 153 & 186 & 90 & 53 \\
\hline$\left|\sigma_{33}^{\max }-\sigma_{33}^{\min }\right|$ in $\alpha$ phase & 21 & 85 & 111 & 136 & 172 & 192 & 103 & 48 \\
\hline
\end{tabular}

Note: $\sigma_{33}^{p}$ is the phase stress signifying stress discrepancy between phases. The subscript 33 denotes the LD of the duplex steel during uniaxial loading and unloading. Unit of stress: MPa. 
during this period of deformation. Small plastic strains, i.e., the $\varepsilon_{33}^{p l}$ of nonzero values, are first identified for the austenite at the load of $500 \mathrm{MPa}$, and for the ferrite at $600 \mathrm{MPa}$, respectively. At $500 \mathrm{MPa}$ which approximately corresponds to the yielding of austenite, the phase stress starts to increase with increasing applied stress. When the load is close to $600 \mathrm{MPa}$, which denotes the deformation stage prior to the yielding of the ferrite, the $\alpha$ phase shows a prominent increase in stress due to its high elastic module. ${ }^{[23,24]}$ Accordingly, this leads to a significant increase in the stress discrepancy between phases. It should be noted that if residual stresses are not negligible in the steel prior to deformation, the development of the phase stress will not be solely associated with the intrinsic elastic/plastic properties of each individual phase.

Different tendencies of the evolution of phase stress are reported in one of our recent works, ${ }^{[15]}$ which indicates that the accommodation of initial (e.g., thermal residual) stress with intrinsic mechanical properties of austenite and ferrite plays an important role in the development of heterogeneous stresses within the duplex steel. On the other hand, during the loading period from 450 to $700 \mathrm{MPa}$, the grain-orientation-dependent stresses are increased due to the preferential yielding of some plastically soft grains in either phases, i.e., the $\{220\}$ and $\{311\}$ grains in austenite and the $\{211\}$ grains in ferrite, as indicated by both the experimental and the modeled response of lattice strains to the applied load. After $700 \mathrm{MPa}$ load when plastic deformation has occurred in all grains of both phases, the overall stresses of austenite and ferrite start to approach each other and a decrease in phase stress is predicted when macroscopic deformation reaches the maximum load of $800 \mathrm{MPa}$. At this stage, the grain-orientation-dependent stresses are significant in both phases due to the strong anisotropy of plastic properties among the different $h k l$ lattice planes. The simulation has shown that, for the $\gamma$ phase, the average stresses of grains with $\langle 110\rangle \gamma / / \mathrm{LD}$ and $\langle 111\rangle \gamma / / \mathrm{LD}$ are much higher than that with $\langle 100\rangle \gamma / / \mathrm{LD}$ and $\langle 311\rangle \gamma / / \mathrm{LD}$. For the $\alpha$ phase, higher stresses are found in the grains with $\langle 111\rangle \alpha / / \mathrm{LD}$ and relatively low strains in the grains with $\langle 100\rangle \alpha / /$ LD. In addition, a more rapid evolution of plastic strain is predicted in the $\alpha$ phase than in the $\gamma$ phase after loading up to $700 \mathrm{MPa}$, indicative of the significant strain hardening of austenite.

The unloading process of the duplex steel is also modeled and the calculated microstresses are listed in Table II. During this period, remained plastic strains of constant values, i.e., 5.97 and 6.09 pct, are predicted for the austenite and the ferrite, respectively, denoting the elastic unloading of each phase. Obviously, both phase stress and grain-orientation-dependent stresses in the respective phases decrease monotonically with the decreasing applied stress, whereas moderate intergranular stresses still exist in each individual phase when the load is completely released.

It is also noted that during the plastic deformation at a peak load of $800 \mathrm{MPa}$, even though the smallest microstresses are not found in the $\langle 311\rangle \gamma / / \mathrm{LD}$ and $\langle 211\rangle \alpha / / \mathrm{LD}$ grains for respective phases as that in the $\langle 200\rangle \gamma / / \mathrm{LD}$ and $\langle 200\rangle \alpha / / \mathrm{LD}$ grains, compressive stresses are predicted in the former couple of grains after the unloading. As given elsewhere, ${ }^{[25]}$ the diffraction elastic constants of $\{311\}$ planes in austenite and $\{211\}$ planes in ferrite are much higher than that of the other $h \mathrm{kl}$ planes in respective phases, and this may explain the rapid release of stresses in both of the lattice planes with decreasing loading. The simulated average stress of the duplex steel and its constituent phases along the LD as a function of macrostrain of the material is shown in Figure 4(b), in which the simulation for the duplex steel excellently captures the measurement obtained by a mechanical test. The stress partition between austenite and ferrite could be clearly identified when the applied stress is higher than $500 \mathrm{MPa}$.

Except for the stress/strain evolution as discussed preceding, the self-consistent model could also predict the deformation mode activity of the material. As shown in the simulated tensile loading of the duplex stainless steel (Figure 5), when analyzing the relative activity of different slip systems in each phase, it is obvious that at the initial stage of deformation $(\varepsilon \sim 0.2 \mathrm{pct})$ there exists a flat region (with zero value) in the relative activity $v s$ macrostrain curve for both $\gamma$ and $\alpha$ phases, indicative of the elastic deformation of respective phases. After that, the dislocation sliding occurs first on the $\{111\}\langle 110\rangle$ systems in austenite. When the macrostrain reaches $\sim 1$ pct, deformation via slip on both the $\{111\}\langle 110\rangle$ systems in the $\gamma$ phase and the $\{110\}\langle 111\rangle$ and $\{112\}\langle 111\rangle$ systems in the $\alpha$ phase become dominant. The number of active systems in grains could be determined by the stress/strain states of the material. As shown in Figure 6, when the macrostrain is below $\sim 0.2$ pct a flat region of the weighed average of the active systems in each grain (AVACS) vs strain curve is identified for both phases, denoting that no dislocation sliding occurs in either of the phases. At the early stage of plastic deformation $(\varepsilon<2$ pct), the AVACS shows a rapid increase in both phases with increasing macrostrain. With the further elevated load, the strain hardening of the $\gamma$ phase could be judged from the

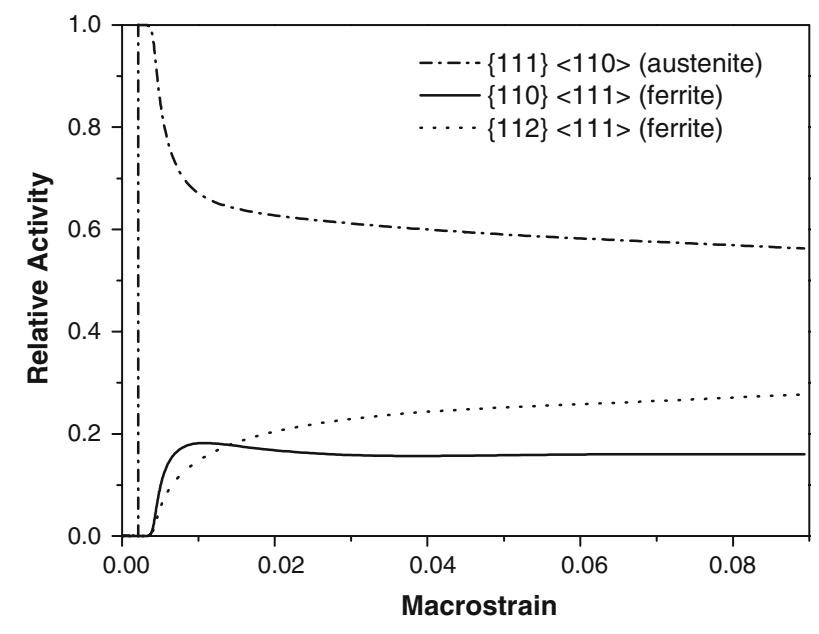

Fig. 5-Self-consistent model calculated relative contribution of slip systems of austenite and ferrite to total shear of duplex steel as a function of deformation. 


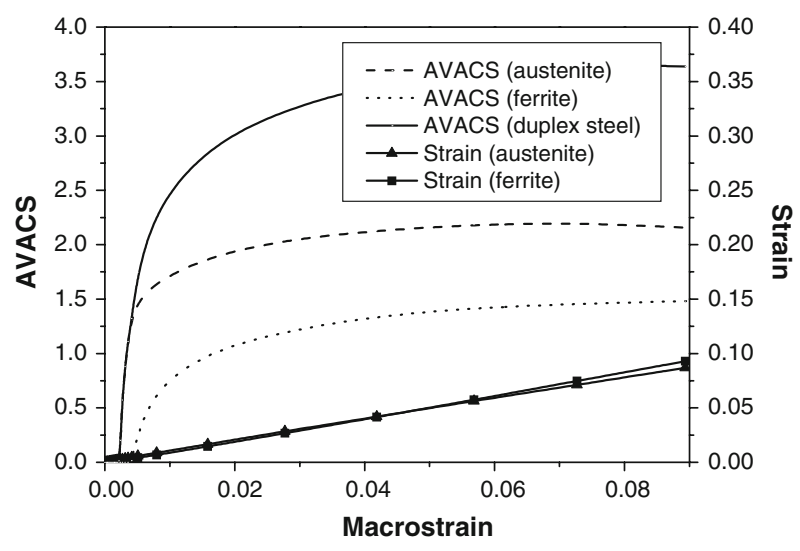

Fig. 6-Self-consistent model calculated average number of AVACS and strain as a function of deformation, for the duplex steel and its constituent phases.

saturation of the AVACS values for the $\gamma$ phase and the concomitant strain compatibility between phases at around 6 pet macrostrain.

\section{SUMMARY}

In-situ neutron diffraction was used to measure the response of lattice strain to the applied stress of an austenite-ferrite steel during tensile loading and unloading. A VPSC model was used for simulating the overall micromechanical behaviors of polycrystalline aggregates and the response of lattice strains. The simulations capture all behavior observed by the experiments except for the $(200) \alpha / /$ TD and (200) $\gamma / /$ TD lattice strains. Through the whole loading process, larger stresses were found in grains with $\langle 110\rangle \gamma / / \mathrm{LD}$ or $\langle 111\rangle \gamma / / \mathrm{LD}$ in austenite and with $\langle 111\rangle \alpha / / \mathrm{LD}$ in ferrite, and the $\langle 100\rangle \gamma / / \mathrm{LD}$ and $\langle 100\rangle \alpha / / \mathrm{LD}$ grains showed smaller stresses. After unloading, compressive stresses were found in the $\langle 311\rangle \gamma / / \mathrm{LD}$ and $\langle 211\rangle \alpha / / \mathrm{LD}$ grains. These are closely related to the elastic and plastic anisotropy of grains having orientations in respective phases. The stress discrepancy between constituent phases of the duplex steel was also calculated with the model. According to the current investigation, an in-depth understanding of the stress heterogeneity in two-phase materials during tensile deformation is obtained.

\section{ACKNOWLEDGMENTS}

This work has been supported by the National Natural Science Foundation of China (Grant Nos. 50671022 and 50725102), the National Ministry of
Education of China (key project), and the Swedish Research Council in the framework of SIDA project (Grant No. 348-2004-3475). This work has benefited from the use of the Lujan Center at Los Alamos Neutron Science Center, funded by the Department of Energy Office of Basic Energy Sciences, and Los Alamos National Laboratory, funded by the Department of Energy (Contract No. W-7405-ENG-36).

\section{REFERENCES}

1. B. Leffler: Stainless Steels and Their Properties, Avesta Sheffield AB Research Foundation, Stockholm, 1996, pp. 30-1.

2. J.W.L. Pang, T.M. Holden, and T.E. Mason: Acta Mater., 1998, vol. 46, pp. 1503-18.

3. J.M. Atienza, J. Ruiz-Hervias, M.L. Martinez-Perez, F.J. Mompean, M. Garcia-Hernandez, and M. Elices: Scripta Mater., 2005, vol. 52, pp. 1223-28.

4. R. Lillbacka, G. Chai, M. Ekh, P. Liu, E. Johnson, and K. Runesson: Acta Mater., 2007, vol. 55, pp. 5359-68.

5. I. Alvarez-Armas, M.C. Marinelli, S. Hereñú, S. Degallaix, and A.F. Armas: Acta Mater., 2006, vol. 54, pp. 5041-49.

6. O. Düber, B. Künkler, U. Krupp, H.-J. Christ, and C.-P. Fritzen: Int. J. Fatigue, 2006, vol. 28, pp. 983-92.

7. Y. Tomota, H. Tokuda, Y. Adachi, M. Wakita, N. Minakawa, A. Moriai, and Y. Morii: Acta Mater., 2004, vol. 52, pp. 5737-45.

8. D.G. Carr, M.I. Ripley, D.W. Brown, S.C. Vogel, and T.M. Holden: J. Nucl. Mater., 2006, vol. 359, pp. 202-07.

9. S. Harjo, Y. Tomota, and M. Ono: Acta Mater., 1998, vol. 47, pp. 353-62.

10. I. Karaman, H. Sehitoglu, H.J. Maier, and Y.I. Chumlyakov: Acta Mater., 2001, vol. 49, pp. 3919-33.

11. D. Dye, H.J. Stone, and R.C. Reed: Acta Mater., 2001, vol. 49, pp. 1271-83.

12. A. Baczmański and C. Braham: Acta Mater., 2004, vol. 52, pp. 1133-42.

13. R. Dakhlaoui, A. Baczmański, C. Braham, S. Wroński, K. Wierzbanowski, and E.C. Oliver: Acta Mater., 2006, vol. 54, pp. $5027-39$.

14. J.W. Hutchinson: Proc. R. Soc. London, 1976, vol. 348A, pp. 10127.

15. N. Jia, R. Lin Peng, Y.D. Wang, S. Johansson, and P.K. Liaw: Acta Mater., 2008, vol. 56, pp. 782-93.

16. M.A.M. Bourke, D.C.E. Dunand, and E. Üstündag: Appl. Phys. A, 2002, vol. 74, pp. 1707-09.

17. A.C. Larson and R.B. Von Dreele: Los Alamos National Laboratory Report LAUR 86-748, Los Alamos National Laboratory, Los Alamos, NM, 2004, pp. 1-224.

18. J. Eshelby: Proc. R. Soc. London, 1957, vol. 241A, pp. 376-96.

19. J.W. Hutchinson: Proc. R. Soc. London, 1970, vol. 319A, pp. $247-$ 72 .

20. U.F. Kocks, C.N. Tomé, and H.R. Wenk: Texture and Anisotropy, Cambridge University Press, Cambridge, United Kingdom, 1998, pp. $467-70$.

21. C. Tome, G.R. Canova, U.F. Kocks, N. Christodoulou, and J.J. Jonas: Acta Metall., 1984, vol. 32, pp. 1637-53.

22. N. Jia, R. Lin Peng, Y.D. Wang, G.C. Chai, S. Johansson, G. Wang, and P.K. Liaw: Acta Mater., 2006, vol. 54, pp. 3907-16.

23. G. Simmons and H. Wang: Single Crystal Elastic Constants and Calculated Aggregate Properties: A Handbook, MIT Press, Cambridge, MA, 1971, pp. 1-370.

24. H.M. Ledbetter: Phys. Status Solidi A, 1984, vol. 85, pp. 89-96.

25. R.L. Peng, Y.D. Wang, G.C. Chai, N. Jia, S. Johansson, and G. Wang: Mater. Sci. Forum, 2006, vols. 524-525, pp. 917-22. 